\title{
CORPUS Corpus
}

Archivos virtuales de la alteridad americana

Vol. 8, No 2 | 2018

Julio / Diciembre 2018

\section{Mujeres afrouruguayas en el contexto del Primer Congreso Nacional de Mujeres del Uruguay (1936)}

Afro-Uruguayan women in the context of the First National Congress of Women of Uruguay

\section{Mónica García Martínez}

\section{OpenEdition}

\section{Journals}

Electronic version

URL: http://journals.openedition.org/corpusarchivos/2613

DOI: $10.4000 /$ corpusarchivos. 2613

ISSN: $1853-8037$

\section{Publisher}

Diego Escolar

\section{Electronic reference}

Mónica García Martínez, « Mujeres afrouruguayas en el contexto del Primer Congreso Nacional de Mujeres del Uruguay (1936) », Corpus [En línea], Vol. 8, No 2 | 2018, Publicado el 19 diciembre 2018 consultado el 01 mayo 2019. URL : http://journals.openedition.org/corpusarchivos/2613 ; DOI 10.4000/corpusarchivos.2613

This text was automatically generated on 1 May 2019. 


\title{
Mujeres afrouruguayas en el contexto del Primer Congreso Nacional de Mujeres del Uruguay (1936)
}

\author{
Afro-Uruguayan women in the context of the First National Congress of Women \\ of Uruguay
}

Mónica García Martínez

\section{Introducción}

1 La colectividad afrouruguaya participó con voz propia en todas las instancias históricas del Uruguay independiente. Incluso desde antes. Durante la época colonial, tuvo sus formas organizativas en el marco del sistema esclavista, ${ }^{1}$ por medio de asociaciones como las naciones, las cofradías, los toques de tambor. Formó sus clubes y su prensa ${ }^{2}$ con el primer periódico La Conservación (1872), en el último cuarto del siglo XIX, cuando Uruguay entraba al periodo de modernización (Méndez 1975), en la primera fase de consolidación del Estado nacional ${ }^{3}$ (Yaffre 2000). Durante este periodo, la prensa, en general, crece de forma notable en el Río de la Plata (Lobato 2009, p. 10) y un incipiente colectivo afrouruguayo produce las primeras publicaciones, a partir de las cuales da comienzo a una opinión pública propia que llega hasta nuestros días. ${ }^{4}$

2 A lo largo del tiempo y en diferentes contextos el movimiento afrouruguayo se presentó en la esfera pública con nombres diversos, que pasaron de "sociedad de color" a "raza negra", para llegar a los días actuales como "afrouruguayos", entre otros términos, lo cual explica en parte la trayectoria de sus procesos identitarios. ${ }^{5}$ (Andrews 2012, pp. 30-31) (García 2018).

3 En la década de los treinta del siglo XX, las mujeres afrouruguayas comenzaron a participar en los movimientos sociales con otras mujeres de su país, como fue el caso del 
Primer Congreso Nacional de Mujeres del Uruguay, realizado en Montevideo en 1936, en el cual representaron a la "Raza Negra" -término de la época-, las delegadas Iris Cabral y Maruja Pereira. ${ }^{6}$

4 Por otra parte, la representación de las delegadas fue entendida por la revista afrouruguaya Nuestra Raza ${ }^{7}$ como una acción de la "mujer negra". Dos redactores de la revista cubrieron la noticia del congreso y por su medio es posible acercarse a la noción de la diferencia entre ser mujer y "mujer negra".

En este artículo se hará una reseña sobre el congreso en general, visto como un espacio de comunidad de mujeres a nivel nacional, principalmente, en el cual estuvieron dos afrouruguayas participando como exponentes y asistentes. Se señalarán, asimismo, otras conexiones de la colectividad afrouruguaya con mujeres organizadas y por último se buscará entender qué se entendió por "mujer negra".

6 Para observar las dos pertenencias - mujer y afrodescendiente-, se utiliza la categoría de interseccionalidad en tanto puede dar cuenta de varios cruces identitarios que son vividos a la vez. La noción de interseccionalidad proviene de activistas afroestadunidenses y fue definida por Kimberle Crenshaw para explicar un fenómeno complejo de desigualdad en el que participan diferentes cruces de inequidad, provenientes de relaciones de dominación, que operan al mismo tiempo. ${ }^{8}$ Es el caso de las mujeres racializadas en tanto "negras". Para Crenshaw, las afrodescendientes viven una estructura de opresión que no puede ser explicada ni en tanto mujeres ni en tanto "negras", por separado. Para la autora, la interseccionalidad, entonces, es una estructura en la que se vive la opresión de forma múltiple, en donde los distintos cruces operan al mismo tiempo.

\section{El contexto del congreso y la colectividad afrouruguaya}

7 Para la fecha de la realización del primer Congreso Nacional de Mujeres del Uruguay en abril de 1936, el momento era difícil. Bélico y totalitario a nivel internacional y represivo a nivel nacional con la dictadura de Gabriel Terra.

8 Las mujeres activistas debían pronunciarse sobre los acontecimientos mundiales y, asimismo, encontrarse y deliberar sobre problemas comunes, denunciar abusos en los entornos en los cuales se movían, reivindicar derechos y proponer estrategias conjuntas. Urgía a partir del encuentro una agenda de planificaciones y acciones.

9 La década de 1930, hondamente influida por la crisis mundial de 1929, trajo cambios importantes a nivel nacional e internacional. El contexto local vivió un viraje hacia el autoritarismo con el golpe de Gabriel Terra, quien trajo una dinámica de represión distinta a la de las décadas anteriores con el batllismo. ${ }^{9}$ Entre otras medidas, el gobierno de Terra impuso el cierre de la Asamblea General, la disminución de proyectos sociales instaurado pocas décadas atrás, censura a la prensa, protestas con detenidos y una política migratoria que cerró las puertas a muchos inmigrantes clasificados como "indeseables", entre otros resultados. Estas medidas represivas sustraían derechos adquiridos años antes, por lo que forzaron a los movimientos sociales a nuevas estrategias.

10 A nivel internacional, tanto en América Latina como en gran parte del mundo se hacía un viraje hacia gobiernos autoritarios: golpes militares en el continente y en Europa, el 
surgimiento del fascismo, del nazismo y del franquismo. La Guerra Civil Española tuvo gran repercusión en el Uruguay y muchas mujeres y hombres de diversos movimientos se organizaron para apoyar los distintos frentes populares organizados en España bajo la guerra. El plano político de tensiones y guerras que estaban agitando Europa tuvo su repercusión en Uruguay y generó un clima de indignación en la opinión pública. Así, se plantearon debates, reflexiones, campos de discusión y movilizaciones tanto en el terreno teórico como en la praxis. Comités contra la guerra y el fascismo hubo en todo el país y también en la región.

11 El movimiento afrouruguayo no fue ajeno. Tuvo vínculos con el movimiento obrero nacional e internacional y rechazó absolutamente cualquier manifestación totalitaria o a favor de la guerra, viéndose interpelado a tomar posición. Para estos años también se había fortalecido de forma importante, del mismo modo que en gran parte del mundo, a consecuencia de un giro significativo hacia lo "negro" que había tomado vigor.

12 Una comunidad internacional "negra", de africanos y afrodescendientes -y de gente que, sin serlo, la apoyó-, se configuró a partir de los años veinte del siglo pasado y actuó artística y políticamente por medio de diferentes movimientos. Entre los más conocidos están: el Renacimiento de Harlem, la Negritud de Aimé Césaire, Léopold Sédar Senghor y Leon Damas, el nacionalismo negro trasnacional de Marcus Garvey, el Frente Negra Brasilera, el marxismo negro de Harry Haywood, C. L. R. James, W. E. B. Du Bois, las grandes movilizaciones por la descolonización de África, entre otros (Lao-Montes 2009, pp. 217-223).

13 Asimismo, para la década de los treinta, las y los afrouruguayos, con experiencia en cuestiones de organización política, se afirman en su negritud y, así como sus pares en el mundo, resignificarán nombres impuestos como "negro" o "raza" dándoles su propio sentido. Estamparán en sus publicaciones, títulos y subtítulos, titulares y proclamas, términos alusivos, como "nuestra raza", "órgano de la raza negra", "partido autóctono negro", etcétera. En esta época también se fortalecen los vínculos entre las organizaciones de la capital y las del interior del país; así como con personas y movimientos en el exterior. Ejemplo de ello fue la inclusión de dos escritores de la prensa afrouruguaya en la monumental antología de la escritora y editora inglesa Nancy Cunard, en 1934: Negro. Anthology: Elemo Cabral con The Negro Race in Uruguay y Marcelino Bottaro con Rituals and Candombes, y de tres trabajos de Pereda Valdés, uruguayo, no afrodescendiente, que se había sumado a las movilizaciones del colectivo y era, asimismo, colaborador de la revista Nuestra Raza.

En miras a organizarse para la acción, dentro de este contexto, la colectividad afrouruguaya formó el "Comité de la Raza Negra contra la guerra y el fascismo", en noviembre de 1935, con la finalidad de manifestar el apoyo a dos fuerzas: por un lado, a Etiopía, invadida por las tropas de Mussolini (Rodríguez 2009) y, por el otro, al pueblo italiano, "la primer víctima de la aventura fascista". ${ }^{10}$ (Nuestra Raza 1935, p. 8). Estas adhesiones demuestran que la solidaridad del colectivo afrouruguayo no era apenas con la comunidad racial mundial, sino con la de los pueblos oprimidos; en este caso el pueblo italiano, "pues el fascismo para ellos significa hambre, miseria, dolor, y carencia total de libertades y la guerra, derramamiento de sangre en holocausto al capricho de un monstruo que impone a millones su voluntad soberana". (Nuestra Raza 1935, noviembre, p. 8).

En una de las actividades del comité se contó con la presencia de Maruja Núñez de Varela que "habló a la concurrencia en nombre de la mujer negra" (Nuestra Raza 1935, diciembre, 
p. 12), lo cual da cuenta ya de la aparición de esta categoría, que pasó a tener sentido en acciones emprendidas dentro de contextos específicos. Es como representantes de la colectividad y de su comité contra la guerra que Iris Cabral y Maruja Pereira comparecen al Primer Congreso Nacional de Mujeres como "mujer negra". La iniciativa de la participación de ambas no fue entonces un acto aislado o personal, sino que correspondió a la representación que las mujeres de la colectividad hacían de la institución creada recientemente por el colectivo afrouruguayo: el "Comité de la Raza Negra contra la guerra y el fascismo". Esta participación, como lo apunta Carrol M. Young, significó un momento de gran importancia para todo el movimiento afrouruguayo (Young 2004).

\section{El Congreso}

Es en este contexto que se reunieron por primera vez las mujeres del Uruguay, durante seis días, del 17 al 23 de abril de 1936, en Montevideo. La voluntad y entusiasmo traspasó las fronteras y llegaron delegaciones de Cuba y Argentina. De este último país lo hicieron provenientes de comités de las ciudades de Buenos Aires, Córdoba, Rosario y Mendoza. El diario El Día hizo una amplia cobertura con abundante información y fotografías. Más de 50 entidades enviaron representación. El vespertino Uruguay daba la noticia el mismo día de la inauguración:

"Estuvieron representadas en el mencionado acto la totalidad de las Asociaciones, Confederaciones y Comités feministas contra la guerra y el fascismo del interior de la República y también representaciones de algunas provincias argentinas y de Cuba, que dieron especial realce al espectáculo." (Uruguay 1936, abril, p. 18).

Organizado por la Unión Femenina contra la Guerra, el acto abrió con las palabras de su presidenta María Isabel Cedro Gilardo con una numerosa concurrencia que incluyó la participación de mujeres latinoamericanas. ${ }^{11}$ Durante los seis días, hubo varias conferencias de mujeres conocidas en la arena pública, como la feminista pionera Paulina Luisi, quien aportó tres de las conferencias. Pero además de estos aportes, fueron 


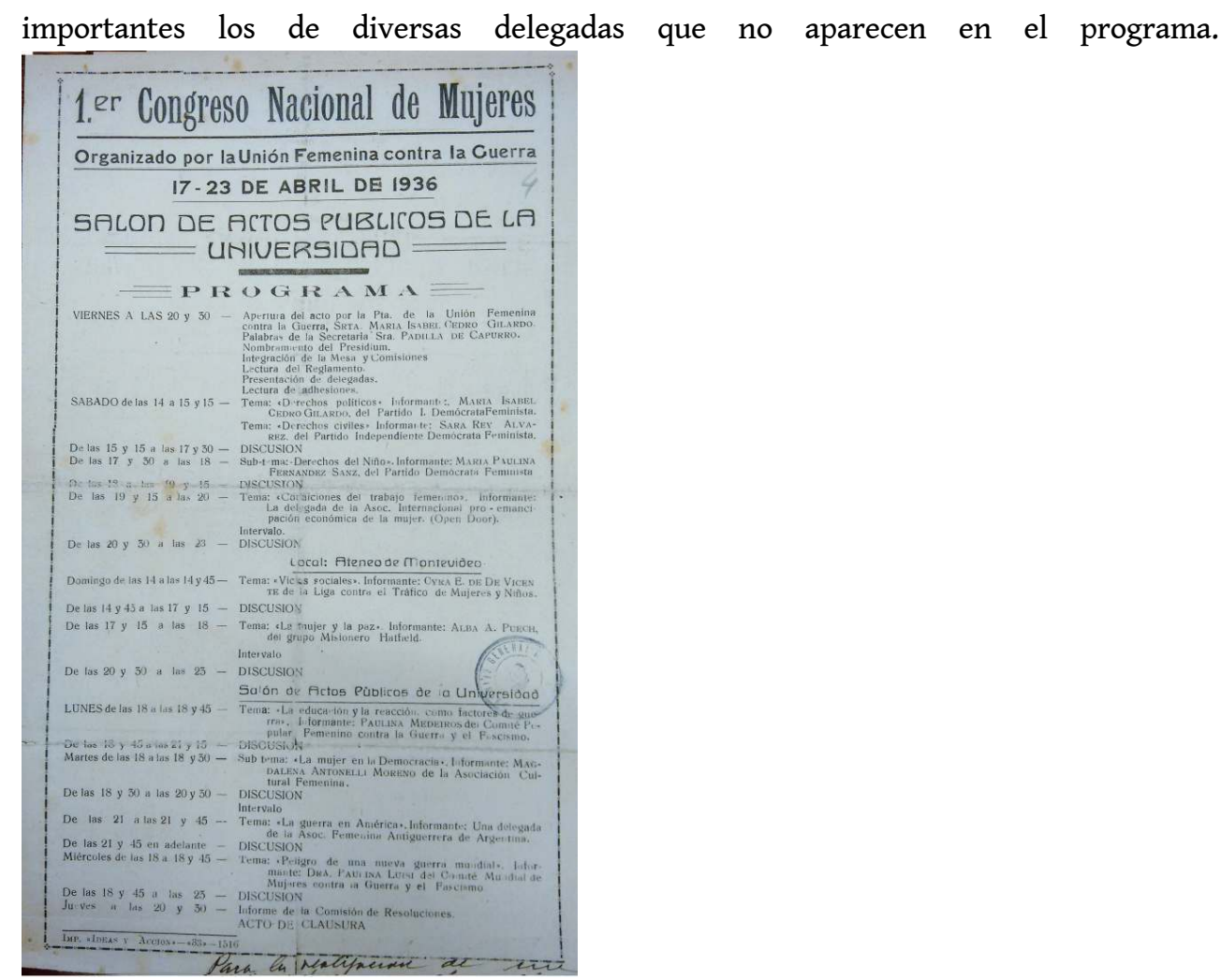

IMAGEN N 1, Programa del Congreso (Archivo de la Dra. Paulina Luisi, Archivo General de la Nación).

17 El motivo principal del congreso era manifestar el repudio a la guerra y ponerse de acuerdo entre las mujeres en esta moción. Sin embargo, sería, a la vez, una excelente oportunidad para tocar otros asuntos importantes a nivel local, que tenían que ver con sus situaciones particulares y, al mismo tiempo exponer algunos puntos de la agenda feminista. De ahí que cada delegada expresó y denunció problemas relacionados con situaciones de sus medios. Se expusieron así, asuntos laborales, falta de protección gubernamental, problemas en la educación, la importancia de la democracia, el rechazo a la guerra, la puntualización de ciertos derechos necesarios para las mujeres, etcétera. Algunas exposiciones entraron en terrenos más filosóficos sobre el género femenino y se refirieron a una especie de "naturaleza" de las mujeres para proponer cuál debía ser la posición a tomar ante la guerra. Una sensibilidad que les concernía en tanto madres, era una característica esencial para repudiar la guerra. Así lo pensó, por ejemplo, la médica recientemente graduada, Juana Amestoy de Mocho, participante del encuentro, quien publica una nota en el diario El Día el mismo martes de la semana del congreso subrayando dos puntos trascendentes del encuentro. Por un lado, la importancia de reunirse las mujeres por primera vez: "nuevo paso que da la mujer del Uruguay, que pondrá en evidencia su preparación y su capacidad (...) capaz de intervenir eficazmente en la solución de todos los problemas que plantee el panorama político y social de nuestro medio". (El Día 1936, abril, p. 17). Y, por otro lado, señalado el rol de madres. Este las colocaba como las enemigas principales de la guerra, ya que eran sus hijos los que marchaban como soldados a arriesgar sus vidas: "parecería que el problema no tuviese solución racional, y que hubiera que esperarla por la vía del sentimiento". (El Día 1936 abril, p. 17). La mujer, por lo tanto, era la más capacitada para imponer el cambio. 
18 Además de las conferencias y participación de las delegadas, hubo propuestas a ser votadas y debates que enriquecieron el encuentro. Debates y demandas discutidos desde hace décadas por las mujeres organizadas fueron puestos nuevamente sobre el tapete, como la igualdad de derechos para mujeres y hombres.

19 Paulina Luisi lo decía con claridad, el objetivo era que "las mujeres de todas las naciones del mundo, disfruten de una igualdad de estatutos y de derechos con los del hombre, en todos los medios de la vida social". (El Día 1936, abril, p. 20). Las concurrentes estuvieron de acuerdo en eso y propusieron más. Se debía: la igualdad de derechos sin importar el estado civil o la edad; acabar con la tutela del marido; la administración de los bienes matrimoniales por parte de ambos cónyuges; que la patria potestad de los hijos fuese de madre y padre; considerar el adulterio de los maridos también como causal de divorcio y no, como hasta el momento, solamente cuando proviniese de las esposas; permitir que las mujeres casadas ejercieran cualquier profesión y concurrir a juicios sin la tutela del marido, entre otras cosas.

20 Las delegadas provenían de diversos ámbitos laborales y sociales: trabajadoras de frigoríficos, tabacaleras, costureras, del servicio doméstico, campesinas, maestras, profesionales, de algunos barrios, de fábricas de calzado, telefonistas, estudiantes y también afrouruguayas, conocidas en la época como representantes de la "Raza Negra". Todas ellas concordaron en el repudio a la guerra y denunciaron problemas a nivel general y de situaciones particulares. Se refirieron a bajos ingresos, problemas con los patrones, despidos por problemas de salud, desalojos por no poder pagar los préstamos bancarios, gran desocupación en el magisterio en contraposición a un alto número de analfabetismo, entre otros asuntos. Para avanzar en los cambios de este panorama se concordó que debían hacerse innovaciones en los códigos jurídicos y, en algunos casos, crearse sindicatos, como en el caso de las trabajadoras domésticas.

21 Las noticias sobre el congreso fueron divulgadas de forma diferente por los medios de prensa, lo que da la idea de las distintas posiciones políticas. Fue difundido como noticia de destaque para algunos, como lo fueron el vespertino Uruguay o el diario El Dia, el cual cubrió el evento con columnas de crónica y opinión de todos los días del congreso, además de un número importante de fotografías. Algunos diarios y revistas apenas lo hicieron, como El País, con un par de párrafos. Otros ni siquiera lo mencionaron, y hubo el que no estuvo conforme con la noticia como fue el caso de El Diario, el cual en una postura conservadora se pronunció tan negativamente hacia las mujeres del congreso que las mismas tuvieron que dedicar un momento de su programa para protestar por lo que consideraron agresión.

22 Entre tanto, la comunidad afrouruguaya, organizada por medio del "Comité de la Raza Negra contra la guerra y el fascismo", estaba familiarizada con el tema de la guerra a través de las reuniones y debates que venía realizando. El congreso de mujeres fue un excelente marco para dar a conocer el colectivo y el trabajo que venía realizando. A su vez, fue un momento idóneo para Iris y Maruja el entablar contacto con otras mujeres de diferentes situaciones.

\section{La “mujer negra” en el congreso, según Nuestra Raza}

23 La revista mensual Nuestra Raza le dedicó dos artículos a la noticia del congreso en dos de sus números, 33 y 34, de abril y mayo de 1936, respectivamente. No era para menos, las 
dos delegadas del "Comité de la Raza Negra" en el congreso de mujeres eran, además, compañeras de trabajo. Iris Cabal se encargaba de organizar la sección social y Maruja Pereira colaboraba con algunos escritos. Ambas, a su vez, eran componentes de grupos de teatro de la colectividad. Nuestra Raza al dar la noticia de la participación de la "mujer negra" puso a la vista algunas de sus características y los motivos de su adhesión al congreso.

Principalmente por una nota del vespertino Uruguay, se sabe que la participación de ambas delegadas se realizó el primer día del congreso el viernes 17 de abril, junto con otras. Según una nota publicada años después por Nuestra Raza, se sabe que la propuesta que presentaron fue relacionada al trabajo doméstico y a la formación de un gremio. ${ }^{12}$

La nota de Nuestra Raza que lo recuerda fue publicada seis años después, en mayo de 1940, y en esa oportunidad daba la buena noticia sobre la formación de una organización gremial que buscaba establecer la jubilación para las y los trabajadores del sector doméstico. La nota agregaba el dato que no se había dado en la época del congreso: cuál había sido la exposición y propuesta de las delegadas Iris y Maruja. Según lo que dijo Nuestra Raza cuatro años después, la ponencia de ambas delegadas se había referido a la necesidad de la formación de un gremio del servicio doméstico. Propuesta pensada y elaborada en el seno del "Comité de la Raza Negra contra la guerra y el fascismo"; es decir no provenía de la "mujer negra", necesariamente, sino en conjunto con su colectividad.

"Gremio que se organiza

Uno de los más numerosos gremios -el del servicio doméstico- el más mal tratado y remunerado de todos, se ha constituído en entidad gremial con el fin de reclamar sus derechos como parte integrante de la sociedad a la que presta su valioso servicio. (...) Acogemos con simpatía este movimiento, no ya tan solo por la justicia que el supone, sino también por que sería algo así como la cristalización de nuestras ideas, por cuanto fue una de las ponencias que presentó el "Comité de la raza negra contra la guerra y el fascismo" por intermedio de sus delegadas Iris Cabral y Maruja Pereira, en el Congreso de Mujeres realizado en Montevideo, en Abril del año 1936 y que fue aprobado por unanimidad por los congresales asistentes." (Nuestra Raza 1940, mayo, p. 3).

\section{Dos artículos sobre “Mujer negra” en Nuestra Raza}

Al no encontrarse información proveniente de las propias delegadas o textos de su participación, se utilizan los comentarios de los cronistas de Nuestra Raza como fuente para entender su papel en el congreso y, a su vez, saber qué entendía la colectividad por "mujer negra" en ese momento, por qué comenzó a adquirir significado, dado que anteriormente a este contexto la expresión no era utilizada. Sin embargo, el término "mujer negra" no aparece en los periódicos hasta Nuestra Raza de la segunda época.

Antes de continuar es importante tomar en cuenta dos aspectos.

Primero. La participación de las mujeres afrodescendientes en su colectividad -aunque no utilizasen el término "mujer negra"-, fue preponderante en todas las organizaciones afrouruguayas a lo largo de su historia (Rodríguez 2006, p. 84) (Andrews 2012, pp. 71-75) (Instituto Nacional de las Mujeres 2011). En las páginas de la prensa afrouruguaya del siglo XIX, por ejemplo, son innumerables las menciones a su participación en la organización de centros femeninos, reuniones, actos, conferencias, bailes, tertulias, rifas, tanto para el sostenimiento de la vida social como para la reunión de fondos para las instituciones de la colectividad (entre ellas las publicaciones). Asimismo, su papel de 
escritoras existía desde el siglo XIX. (García 2014, pp. 115-142). De destaque es la escritura de María Esperanza Barrios y Margarita Ubarne Mansilla como columnistas. La primera como editora y escritora de la primera época de Nuestra Raza, en San Carlos, Maldonado, en 1917. La segunda en La Propaganda (1911-1912) y La Verdad (1911-1914) como columnista y poeta, y con colaboraciones en La Vanguardia (1928) y Nuestra Raza (1934). Segundo. El término "mujer negra" para autonombrarse públicamente perduró. Además de seguir colaborando para Nuestra Raza, fue muy importante, a un año después del congreso, (exactamente a partir de abril de 1937), el papel de redactoras de dos mujeres afrouruguayas, María Felina Díaz y Maruja Pereyra, en los nueve números de la publicación periódica del Partido Autóctono Negro (P. A. N.), con la dirección de Sandalio del Puerto, expresando sus ideas de cuáles debían ser las acciones estratégicas a seguir de la "mujer negra", principalmente para las elecciones de 1938. Una de estas acciones fue la formación del Comité femenino de mujeres afrouruguayas en Rivera (P.A.N. 1937, diciembre, 17, p. 3). Más adelante el término "mujer negra" sigue siendo utilizado para autonombrarse (Instituto Nacional de las Mujeres 2011). Para 1942, por ejemplo, se encuentra en la proclama "Las mujeres negras a sus hermanas de todo el país", firmada por cien mujeres, por motivo de los festejos del centenario de la abolición de la esclavitud (Nuestra Raza 1942, noviembre, p. 7). Todo lo cual demuestra que, luego del congreso, las acciones públicas de las afrouruguayas fueron realizadas desde la "mujer negra". ${ }^{13}$

Tomando en cuenta estas consideraciones, se analizarán las dos notas con que Nuestra Raza cubrió la noticia del congreso.

Primera nota:

"La mujer negra que pagando tributo a los resabios del medio ambiente más que por su natural apatía, mucho más que por propia despreocupación, ha vivido aislada, desconocida en el ambiente nacional hasta hoy, que llevada por la sana influencia de las nuevas corrientes sociales y políticas que van dejando atrás, simplificando hasta anular diferencias y odios raciales, interviene por primera vez en la dilucidación de un problema universal, confundida con su congénere la mujer blanca, de todas las ideologías y de todas las capas sociales que piensan, sienten y sufren el dolor y las consecuencias de ese mal que amenaza seguir azotando a la humanidad: la guerra." (Nuestra Raza 1936, abril, p. 1).

A partir de la primera nota se pueden observar tres sentidos: la diferencia con las demás mujeres, su representatividad, algunas características y su aparición política en el medio público. La diferencia con otras mujeres es perceptible desde que la "mujer negra" actuó en el congreso en un espacio de mujeres en general, sus "congéneres" entendidas como "blancas". Su representación no es de la "mujer negra" en sí, sino de la colectividad afrouruguaya que por medio de sus mujeres tiene voz en un congreso de mujeres. Es decir, la voz no era de las mujeres afrouruguayas, solamente, era de su colectividad.

Algunas características que el cronista señala para la "mujer negra" son el resabio y la apatía, explicados históricamente:

Las expresiones utilizadas por el redactor "apatía y despreocupación" corresponden a lo que la colectividad afrouruguaya en general opinaba sobre sí misma -y no necesaria o únicamente sobre las mujeres del grupo-. Estos juicios se acompañaban de otros también negativos como "desidia", "falta de interés", "alejamiento". Casi siempre estas acepciones se ponían sobre el tapete en un sentido autocrítico cuando el discurso hacia la 
colectividad apelaba a la unión. Lo único que impedía dicha unión era la "apatía", la "falta de interés", etcétera, de los miembros de la colectividad. ${ }^{14}$

"Paga tributo por el medio y sus resabios". Aquí, también tiene que ver con la colectividad y no solamente con las mujeres del grupo. Se hace referencia a la histórica condición de relegación a la que sus antepasados fueron sometidos por la esclavización. La "apatía" tiene, por lo tanto, otra causante: la esclavización de sus abuelos, la cual les había impedido tomar las riendas de sus vidas. Al referirse a esta etapa se agregaba, en ocasiones, que felizmente ya había quedado atrás, por lo que la gente de la comunidad solamente debía esforzarse para "regenerarse", ya que los tiempos de látigos habían pasado. $^{15}$

"Es desconocida en el ambiente nacional". Este es un aporte realmente nuevo en las reflexiones de la comunidad: las mujeres afrouruguayas eran desconocidas, no habían participado organizadas en la escena pública.

El momento también era determinante; 1936 fue un año en el que se esperaba que las mujeres votasen en las siguientes elecciones, las de 1938, la ley del voto femenino había sido aprobada en $1932 .{ }^{16}$ Sin embargo, no parecía ser algo seguro. En noviembre de 1937, María Felina Díaz en su columna desde el órgano del P.A.N. cuestionaba la posibilidad de que el sistema electoral no permitiese aún la aplicación de la ley del voto femenino. ( P.A.N. noviembre, 1937, p. 3). En cambio, si lograban votar, entonces significaba un importantísimo caudal de votos que debían ser tomados en cuenta y disputados por los diferentes partidos. La propia colectividad afrouruguaya estaba formando su propio partido, el Partido Autóctono Negro, y las mujeres, en el caso de formar parte del electorado, serían claves para aumentar el número de votos.

Otra reflexión del redactor es por qué participaron del congreso:
“¿Por qué esa intervención: Porque frente al anhelo humano que sirve de eje motor a este Congreso, la mujer negra que integra la sección femenina del Comité de la Raza Negra contra la guerra y el fascismo, constituido también por hombres de todas las ideologías, que representan la mayoría de una colectividad que prejuiciada o no, forma parte del conglomerado nacional, sola condición ésta que la habilita perfectamente para intervenir en todos los problemas que atañen directa o indirectamente al pueblo." (Nuestra Raza 1936, abril, p. 1).

La colectividad afrouruguaya en vías de pronunciar su candidatura para representantes nacionales en las elecciones por venir con el recién creado Partido Autóctono Negro y de cara a un contingente de personas afrodescendientes en todo el Uruguay, de distintas corrientes políticas - tanto de los partidos tradicionales, el Blanco y el Colorado como corrientes de izquierda $u$ otras no representadas en las elecciones-, debía poner en consideración este abanico político a la hora de organizarse estratégicamente para las elecciones. Por lo tanto, los discursos estuvieron influidos por estos objetivos. La unidad era fundamental, por eso se remarcaba el valor de la organización del "Comité de la Raza Negra contra la guerra y el fascismo" y la importancia del ejercicio de la ciudadanía: "Colectividad prejuiciada o no, forma parte del conglomerado nacional". Esto iba también en relación a su invisibilización como grupo y a la discriminación social. A pesar de todas las circunstancias, las y los afrouruguayos sostenían el derecho a tener derechos en tanto ciudadanos.

La "mujer negra" también se definió a la hora de tomar posición sobre determinados asuntos. Con relación a la guerra en Europa, tiene su postura política: la solidaridad con Etiopía invadida por los fascistas de Mussolini. La "mujer negra" en tanto mujer, de forma 
similar a lo que se vio antes con las delegadas del congreso, tanto madres o hermanas de las víctimas y soldados, debían ser las primeras en repudiar la guerra.

"Porque en estos momentos que el pueblo libre de Etiopía ve arrasar sus ciudades y pierde sus hombres, sus mujeres y sus niños en una infame guerra de conquista conque una de las grandes potencias pretende civilizarlo - con gases venenosos - la mujer negra del Uruguay negaría su condición de tal si como madre o como hermana rehuyera intervenir en un congreso donde el problema central a tratarse es la adversión a la guerra." (Nuestra Raza 1936, abril, p.1).

Le correspondía, entonces, participar del congreso y solidarizarse con sus congéneres. El cronista cierra la nota felicitando a las organizadoras de dicho congreso y a las delegadas del colectivo por su participación "en esta nueva ruta de acercamiento que expusieron las delegadas del “Comité de la Raza Negra”".

En el siguiente número de Nuestra Raza, mayo de 1936, un artículo de Don Nadie de la sección "Desde mi rincón" también comentaba la participación de las delegadas Iris y Maruja como testigo de varias sesiones a las cuales concurrió. Don Nadie agregaba algunos puntos de interés para entender los dos aspectos planteados: sobre quién era la "mujer negra" y por qué participó del congreso:

"Como es del dominio de todos, al referido Congreso asistió, en representación del Comité de la Raza Negra contra la guerra y el fascismo, la mujer negra, que en su condición de mujer del pueblo; que siente y sufre las consecuencias del privilegio hecho dogma, expuso en nombre de la colectividad que representaba, sus puntos de vista concretos, en lo referente al fin primordial que alli la llevaba: la guerra y el facismo." (Nuestra Raza 1936, mayo, p. 3).

La "mujer negra" era aquella perteneciente al pueblo y que "siente y sufre las consecuencias del privilegio hecho dogma". Hay implícita una realidad de clase, en tanto pueblo, en un juego de relaciones de poder históricamente constituidos de los cuales la "mujer negra" era también consecuencia y resistencia.

Su participación en el congreso correspondió entonces a dos pertenencias o identidades. Por un lado, ser parte de la "colectividad que representa" y, por el otro, su alianza natural con sus "congéneres". Desde ambas pertenencias, ser mujer y ser afrodescendiente se pronunciaba contra la opresión, la violencia, la muerte que aparejaba la guerra.

Como pudo verse, la participación estuvo expuesta y comentada por redactores varones de Nuestra Raza, algo que las delegadas como colaboradoras de la revista podrían haber hecho. Esto último, sumado a la lectura de la revista completa y de otras publicaciones de la colectividad, parece mostrar que las mujeres afrouruguayas en ese momento no hablaban completamente con una voz propia, en tanto mujeres, dentro de su colectividad; su papel de "mujer negra" apareció cuando hubo que presentarse hacia afuera. Es decir, para 1936, tuvo participación política como representante de su colectividad, pero no con una identidad y movimiento autónomo, lo cual se verá con mayor autonomía para 1937. Todo lo cual parece indicar que el congreso fue el momento político que dio origen al término. Por otra parte, era como afrodescendientes, como "raza negra" que se habían organizado públicamente. Lo de "mujer", venía después. La participación en el congreso, por lo tanto, fue una nueva forma de activismo en el que se conformaba la intersección mujer y afrodescendiente.

48 Este encuentro, un gran logro de las mujeres uruguayas impulsado por iniciativa de las feministas, era una nueva puerta que se abría para todas. Sin embargo, no deja de observarse que, aunque era para todas, pareciera ser que no existía un mismo nivel de importancia entre ellas. El congreso contó con varias delegadas y varios días para 
exponer y, sin embargo, casi todas expusieron el primer día dejando el resto de la semana para las conferencias magistrales de las mujeres académicas y de las que habían organizado el congreso. Si se observa el programa de la imagen número uno, podrá verse que no se mencionan las exposiciones de las afrouruguayas, ni de las delegadas campesinas, ni las de los frigoríficos, etcétera, sino que se las agrupa a todas como “delegadas". El vespertino Uruguay sí las mencionó y las llamó "oradoras", pero les otorgó apenas un párrafo. En esta diferencia entre mujeres podrían imaginarse situaciones jerárquicas en las que no estaría excluida la relación de empleadas y patronas.

En este sentido, es pertinente observar la historicidad de las diferencias entre las mujeres afrodescendientes y las demás. Es decir, a pesar que compartieron con las demás la misma "construcción política del control al que están sometidas socialmente" (Castañeda 2011, p. 33), jugando en los roles de la esfera conocida como privada, las afrodescendientes presentaron diferencias con las demás mujeres, sobre todo por su acentuado papel en el mundo del trabajo, entre otros aspectos. Históricamente, las mujeres afrodescendientes no existen fuera del mundo laboral, el cual las llevó tempranamente a participar de espacios dentro y fuera de las casas desde la época esclavista en sus diversas funciones de amas de leche, niñeras, cocineras, limpiadoras, costureras, curanderas, parteras, planchadoras, lavanderas, vendedoras, etcétera. No es el caso de las mujeres blancas, las cuales ocupaban el ámbito doméstico por excelencia, y solamente a partir de la sociedad industrial comenzaron algunas de ellas a trabajar fuera de él.

De todas formas y a pesar de esta diferenciación entre mujeres, no dejó de ser una oportunidad de visibilización de las afrouruguayas y de su posición política, no solamente ante la guerra, sino sobre los derechos laborales en el trabajo doméstico y la acción emprendida para la formación de un sindicato para sus integrantes. A tal punto fue importante que generó, como se vio, la afirmación del concepto "mujer negra".

51 Por otra parte, quedó también definido otro cruce en la interseccionalidad de "mujer negra" en los casos de Iris y Maruja: su posición de trabajadoras comprometidas con el trabajo doméstico. Todo lo cual muestra una "mujer negra" que participaba públicamente de tres identidades: afrodescendiente, mujer y clase trabajadora.

Hasta aquí se vieron algunas consideraciones de lo que se entendió que era ser "mujer negra" para el caso de Iris y Maruja en su estreno como participantes en el dominio político. A seguir, se exponen otras conexiones entre las afrouruguayas y otras mujeres organizadas de la sociedad lo cual revela con mayor claridad la acción que las relacionó.

\section{Otras conexiones públicas de las afrouruguayas}

Una participante del congreso se había tornado, meses atrás, colaboradora de Nuestra Raza . Exactamente desde la formación del "Comité de la Raza Negra contra la guerra y el fascismo" a fines de 1935. Se trata de Delfa Boatti, figura que era conocida dentro del feminismo y el anarquismo. Militante anarquista, italiana, cofundadora del centro femenino Emancipación junto con María Collazo. También cofundadora del anarquista Centro Internacional de Estudio Sociales, con María Collazo y otras figuras como Florencio Sánchez, María Casal o Ángel Falco (Jung 2006, p. 43). Delfa, enterada de la creación del comité afrouruguayo contra la guerra, le escribió un artículo a la revista Nuestra Raza expresándoles su simpatía: "De una mujer italiana. Contra la guerra" (Boatti 1936, 5). 

partir de esta colaboración en la revista, lo siguió haciendo por años con artículos, poemas, cuentos. Y cuando la muerte se llevó a la joven Iris Cabral, Delfa escribió un texto expresando su dolor ante lo sucedido y mencionando el congreso. Este mismo texto para Iris fue incluido por Delfa en su libro "Epitafios", publicado casi dos décadas después, dejando entrever hasta qué punto su involucramiento con la comunidad afrouruguaya fue relevante:

"Hacía mucho tiempo que anhelaba estrechar lazos fraternales con la gente de color (...) Fué allí, en ese primer Congreso (...) que mis ojos se humedecieron con una lágrima sincera al oír las palabras de honda tristeza, de humildad y de emoción que Iris Cabral, pronunció con la adhesión para la paz mundial". (Boatti 1937, p. 5).

esa misma época, pocos meses después del congreso, otra mujer afrouruguaya aparece en Nuestra Raza conectada con mujeres activistas. Se trata de Clementina Gómez, profesora de música, intérprete, compositora, fundadora de la Asociación Uruguaya de Músicos. Se destacó por su trabajo en la presentación de conciertos, en la enseñanza musical y también como creadora. Nuestra Raza en varias ocasiones hizo mención a su trabajo. Su vínculo con otras mujeres lo tuvo como integrante del Partido Demócrata Feminista, de la música de cuyo himno fue autora. De este último hecho Nuestra Raza hizo referencia:

"Muy honroso

La Srta Clementina Gómez Fernández, ha compuesto para el Partido Demócrata Feminista, un himno, delicada partitura que ejecutó en compañía de sus discípulos en la noche del día 6 del corriente en los salones del Palacio Salvo." (Nuestra Raza 1936, octubre, p. 3).

La revista también publicó una carta de sus
felicitaban por la calidad musical de su trabajo:

"Estimada correligionaria:

En nombre del Comité Ejecutivo del Partido y de los afiliados en general, cumplimos con el grato deber de dirigirle la expresión de nuestras calurosas felicitaciones por la hermosa música escrita por usted para glosar dignamente las estrofas del himno de nuestro Partido." (Nuestra Raza 1936, octubre, p. 3).

57 El vínculo de Clementina con las mujeres del partido muestra otros lazos de la red de relaciones que se tejían entre las mujeres de diferentes grupos organizados en la década de 1930. Por otra parte, el Partido Feminista, así como también el Partido Autóctono Negro, ingresarían en las elecciones de 1938. Clementina fue otro ejemplo de persona en situación de interseccionalidad, afrouruguaya, feminista y trabajadora con su realidad de profesora de música.

Dos años después, una nota conectaba a Clementina con Delfa Boatti. Esta última escribe una nota aprobando la huelga de músicos. Luego de la nota, denuncia un caso de discriminación hacia Clementina. Según lo que dice el texto, un miembro del Centro Napolitano -el señor Papariello-, había rechazado la actuación de Clementina dentro de una orquesta que tocaría en los bailes del centro. Tuvo que intervenir el presidente de la institución para que la volvieran a aceptar. La reflexión de Delfa y la del presidente Fernández apuntan a que, considerando que la artista era afiliada al centro Napolitano, había atrás un acto de discriminación del señor Papariello hacia Clementina por ser mujer. 
59 En esta última situación no se observa una discriminación hacia Clementina por ser afrodescendiente, sino solamente por su género. Sin embargo, no debería descartarse esta última realidad.

60 Estos casos citados son evidencia de que, en la década de los treinta del siglo XX, las mujeres afrouruguayas a nivel político se organizaban con otras mujeres del país. En el caso del congreso, actuando más como conectoras de su colectividad con otros grupos con los que compartían intereses, que como activistas feministas. A diferencia de la situación 
de Clementina, la cual compartía como igual, como correligionaria con las mujeres del Partido Feminista.

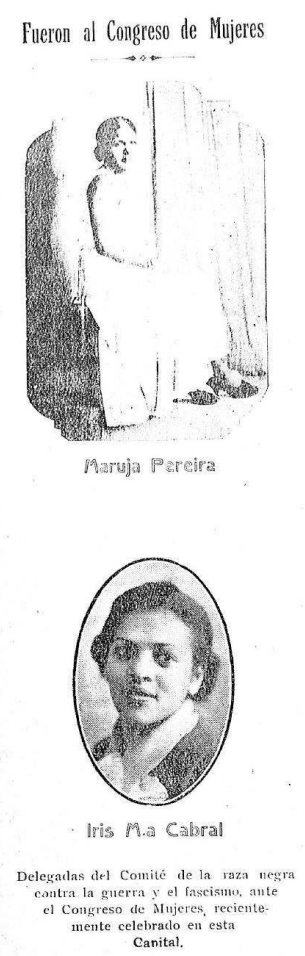

IMAGEN N² 2, Las delegadas, Maruja Pereira e Iris Cabral (Nuestra Raza 1936, abril, p. 6).

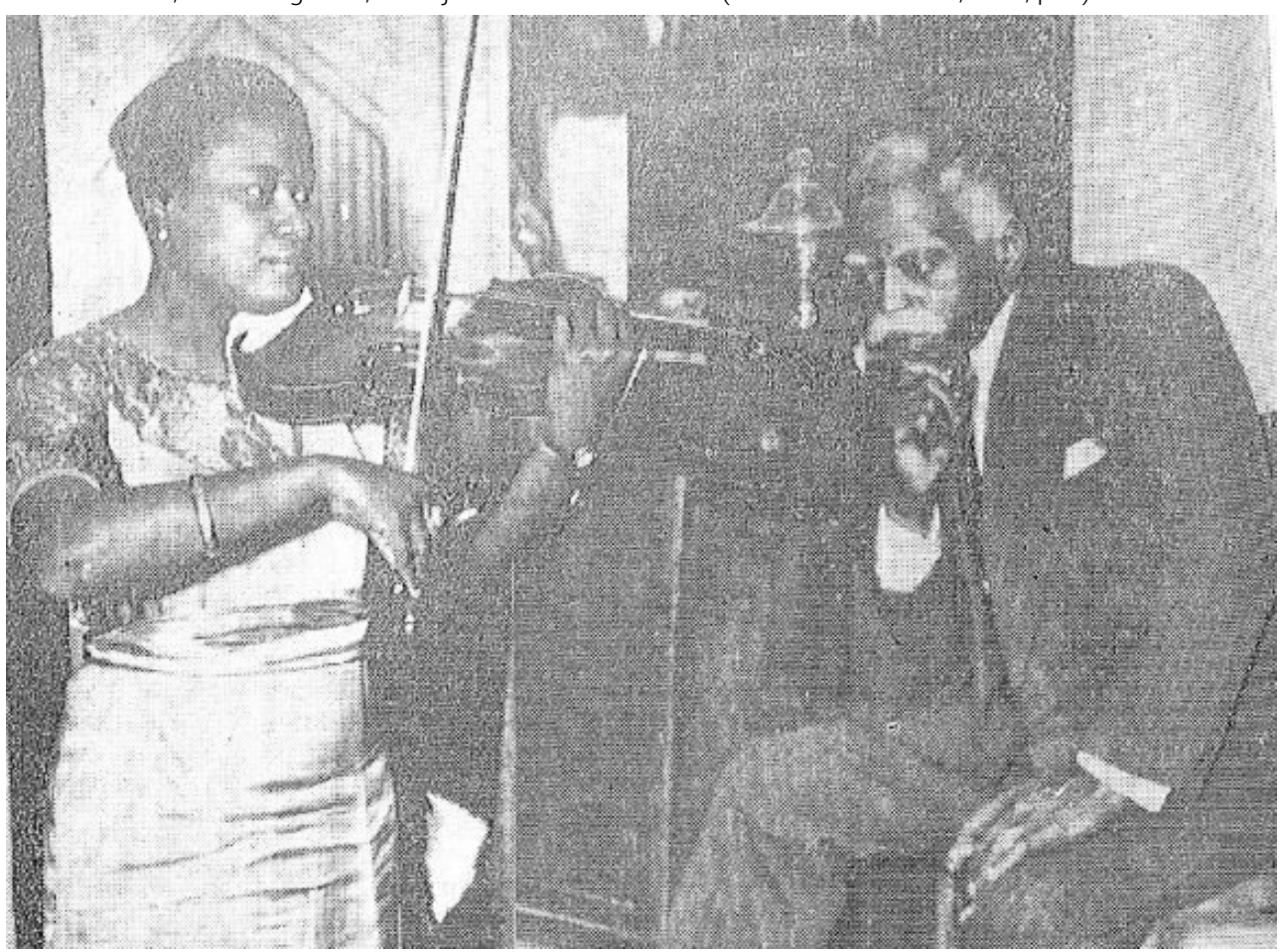

IMAGEN N³, La compositora y profesora, Clementina Gómez (Nuestra Raza 1935, octubre, p. 2). 


\section{Algunas conclusiones}

61 El contexto definió de forma significativa los nuevos movimientos del colectivo afrouruguayo. Dentro de este contexto, en el congreso de mujeres, convergieron fuerzas que influyeron tanto en el colectivo como en las mujeres organizadas del Uruguay, generando una apertura para la aparición pública de las mujeres afrouruguayas, las cuales aparecen como "mujer negra".

A nivel local, el clima dictatorial en el Uruguay y las próximas elecciones con la incipiente participación del Partido Autóctono Negro y el Partido Demócrata Feminista definían estrategias políticas a seguir para diversas fuerzas políticas, todo lo cual facilitó el diálogo entre mujeres, el colectivo afrouruguayo, generando la participación de un nuevo sujeto representado por Maruja Pereira e Iris Cabral: la "mujer negra".

Asimismo, la acción de Clementina Gómez participando del Partido Demócrata Feminista en su calidad de integrante y de artista muestra otra forma en que las mujeres afrouruguayas actuaban políticamente.

A las tres afrouruguayas expuestas, Iris Cabral, Maruja Pereira y Clementina Gómez se las observa en otro cruce interseccional, en tanto trabajadoras, lo cual las colocó como mujeres, afrodescendientes y trabajadoras.

Las participaciones de las afrouruguayas en el congreso y de Clementina en calidad de miembro del Partido Feminista y compositora del himno del partido fueron contadas en su prensa por las voces de los varones de su comunidad, lo cual llevaría a pensar que para ese momento el papel de las mujeres del colectivo estaba aún, en gran medida, mediado por los discursos de los hombres. Sin embargo, como un año después, María Felina Díaz y Maruja Pereira desde las columnas del P.A.N. escribían como "mujeres negras", es posible entender que no solamente el término adquirió fuerza en poco tiempo, sino la propia escritura sobre sí mismas tuvo significativo avance en su autonomía. Y es justamente a partir del contexto del congreso de 1936 que se observan estos cambios.

El testimonio de Delfa Boatti, no afrodescendiente, en su relación con Iris Cabral y en tanto colaboradora de Nuestra Raza, muestra distintos vínculos de mujeres organizadas en el Uruguay, los cuales configuraron redes por las que circulaban informaciones y se generaba sociabilidad.

67 De gran relevancia son las demandas que plantearon Iris y Maruja en el congreso, las cuales apuntaban a una mejor calidad de vida y a la organización de las y los trabajadores del servicio doméstico, evidentemente un espacio donde muchas afroruruguayas se desempeñaban como trabajadoras. Asimismo, la propuesta finalmente dio frutos, por lo que la acción de la "mujer negra" tuvo repercusión.

La "mujer negra" emergió fuera de su colectividad, a nivel público, siguiendo la historicidad, en la intersección de variables que la interpelaban en diferentes ámbitos de la sociedad en general, emitiendo opiniones de la colectividad afrouruguaya al participar del conglomerado nacional. Pero, aunque su principal función fue la representatividad de su colectividad, se generó su necesidad de responder y tomar posición ante problemas internacionales y nacionales como mujeres, como afrouruguayas y como trabajadoras. 


\section{Fuentes}

Archivo de la Dra. Paulina Luisi, Correspondencia particular, 1936-1938, caja 250, carpeta 9, Archivo General de la Nación, Uruguay.

\section{BIBLIOGRAPHY}

Andrews, G. R. (2011). Negros en la nación blanca: historia de los afro-uruguayos 1830-2010. Montevideo: Linardi y Risso.

Boatti, D. (1936). De una mujer italiana. Contra la guerra. Nuestra Raza, Montevideo, febrero, 31, 3-4.

Boatti, D. (1937). Iris M. Cabral. Nuestra Raza, Montevideo, noviembre, 52, 5-6.

Boatti, D. (1938). La huelga de Músicos. Nuestra Raza, Montevideo, septiembre, 61, 10-11.

Boatti, D. (1954). Epitafios. Montevideo: Vértice.

Bracco, R., López, J., Orrego B., Batalla, N. y Bongiovanni, R. (2012). Esclavitud y

afrodescendientes en Uruguay. Una mirada desde la antropología. Montevideo: Facultad de Humanidades y Ciencias de la Educación, Universidad de la República. [Online]. Disponible en: http://www.comisionunesco.mec.gub.uy/innovaportal/file/29929/1/

esclavitud_y_afrodecendientes_en_uruguay.pdf.

Castañeda, P. (2011). Perspectivas metodológicas feministas para el estudio de la identidad de género. En L. Loeza, y P., Castañeda (Coord.), Identidades: teorías y métodos para su análisis (pp. 29-42). México: Universidad Nacional Autónoma de México.

Crenshaw, K. (1991).Mapping the Margins: Intersectionality, Identity Politics, and Violence against Women of Color. [Online]. Disponible en: https://www.racialequitytools.org/.../mappingmargins.pdf.

Díaz, F. (1937). ¿Vota la mujer?. P.A.N., noviembre, 16, p. 3.

Frega, A., Borucki, A., Chagas, K. y Stalla, N. (2004). Esclavitud y abolición en el Río de la Plata en tiempos de revolución y república. En H. N. van Hooff, La ruta del esclavo en el Río de la Plata: su historia y sus consecuencias. Memoria del simposio (pp. 115-147). Montevideo: UNESCO. [Online]. Disponible en: unesdoc.unesco.org/images/0015/001509/150922s.pdf.

García, M. (2014). La identidad afrodescendiente en los periódicos de Montevideo y Buenos Aires a fines del siglo XIX. Tesis de Maestría. Universidad Nacional Autónoma de México, México.

García, M. (2018). Autodesignaciones de las y los afrouruguayos en su prensa (1872-1952).

Intellèctus, 17 (1), Rio. UERJ. [En línea]. Disponible en: https://www.e-publicacoes.uerj.br/ index.php/intellectus/article/view/36012, pp. 1-27.

Instituto Nacional de las Mujeres (2011). Mujeres Afrouruguayas. Raíz y sostén de la identidad. Montevideo: Instituto Nacional de las Mujeres, MIDES. 
Jung, M. E. y Rodríguez, U. (2006). Juan Carlos Mechoso: anarquista, Montevideo: Trilce.

Lobato, Z. (2009). La prensa obrera. Buenos Aires y Montevideo 1890-1958. Buenos Aires, Edhasa.

Lao-Montes, A. (2009). Cartografías del campo político afrodescendiente en América Latina. Universitas Humanística, Pontificia Universidad Javeriana, julio-diciembre, 68, 217-223.

Mbembe, A. (2016). Crítica de la razón negra. Ensayo sobre el racismo contemporáneo, trad. Enrique Schmukler. Barcelona: NED Ediciones.

Méndez, E. (1975). El Uruguay de la modernización 1876-1904. Montevideo: Ediciones de la Banda Oriental

Rodríguez, A. (2009). Un pequeño lugar bajo el sol. Mussolini, la conquista de Etiopía y la diplomacia uruguaya. 1935-1938. Montevideo: Ed. Banda Oriental.

Rodríguez, J. (2006). Mbundo Malungo a Mundele. Historia del Movimiento Afrouruguayo y sus Alternativas de Desarrollo, Montevideo: Rosebud,

Rodríguez, S. y Sapriza, G. (1984). Feminismo y política. Un análisis crítico del proceso de aprobación del voto femenino en el Uruguay. Hoy es Historia, 1 (4), 16-31.

Valdés, T. (2000). De lo social a lo político: la acción de las mujeres latinoamericanas. Santiago de Chile: Lom Ediciones.

Yaffre, J. (2000). Política y economía en la modernización: Uruguay 1876-1933. Primeiras Jornadas de História Regional Comparada, Porto Alegre, agosto. [En línea] Disponible en: www.fee.tche.br/ sitefee/download/jornadas/1/s10a2.pdf.

Young, C. M. (2004). From Voicelessness to Voice: Womanist Writers of the Black Uruguayan Press. Afro-Hispanic Review, 23 (2), 33-38.

Publicaciones periódicas

El Día 1936, Montevideo, abril, 17.

El Día 1936, Montevideo, abril, 20.

La Conservación 1872, Montevideo, octubre, 6.

La Propaganda 1911, Montevideo, agosto, 10.

La Propaganda 1911, Montevideo, junio, $1^{\circ}$.

La Verdad, 1914, Montevideo, mayo, 15.

Nuestra Raza 1935, Montevideo, diciembre.

Nuestra Raza 1935, Montevideo, noviembre.

Nuestra Raza 1936, Montevideo, abril.

Nuestra Raza 1936, Montevideo, mayo.

Nuestra Raza 1936, Montevideo, octubre.

Nuestra Raza 1940, Montevideo, mayo.

P.A.N. 1937, Montevideo, abril-diciembre.

Rumbos 1939, Rocha, junio.

Uruguay 1936, Montevideo, abril, 18.

Ponencia 
García, M. (2017). "Margarita Ubarne Mansilla y sus textos en la prensa afromontevideana". Ponencia presentada en las II Jornadas de literatura y arte afro, Montevideo, julio 2017.

\section{NOTES}

1. El sistema esclavista en lo que hoy es Uruguay existió desde el poblamiento colonial del territorio de la Banda Oriental. A partir de la fundación del primer poblamiento, Colonia del Sacramento, por parte del Imperio portugués en 1680, las primeras personas esclavizadas de origen africano comenzaron a llegar al territorio como "piezas" de la trata esclavista con destino principalmente a la ciudad de Buenos Aires. En la administración hispánica, luego de la fundación de Montevideo, en 1726, los cabildantes de la ciudad solicitaron a la Corona española la introducción de personas esclavizadas de origen africano, las cuales comenzaron a ser traídas a partir de 1743. El final del sistema esclavista en el territorio se dio de forma gradual desde antes y después del Uruguay independiente con varias declaraciones de abolición: 1813 (Junta de Mayo), 1825 (Declaración de Florida), 1830 (Primera Constitución del Estado de la República), 1842 durante la Guerra Grande, (Gobierno de la Defensa, en Montevideo) y en 1847 (Gobierno del Cerrito). Finalmente, en 1862, queda legalmente abolida la última modalidad que permitía la sujeción de personas: el patronato (Frega et al. 2004, pp. 114-145; Bracco et al. 2012, pp. 15-31).

2. Sobre los primeros clubes y publicaciones afrouruguayas, ver: Rodríguez 2006, pp. 56-63; Andrews 2011, pp. 66-75.

3. Jaime Yaffre analiza las dos fases de la modernización. La primera, militarista, en el último cuarto del siglo XIX, en la cual se logra la primera cohesión del país bajo el autoritarismo, y la segunda, entre 1903 y 1933, en que se consolida el Estado de base social (Yaffre 2000).

4. Sobre pasajes de la historia de la prensa afrouruguaya del siglo XIX, ver: Rodríguez 2006, pp. 57-63; Andrews 2012; García 2014.

5. Sobre la significación de las autodesignaciones en la prensa afrouruguaya, ver García 2018.

6. Sobre la participación de Iris y Maruja en el congreso, ver: Young 2004; Rodríguez 2006, pp. 120-121.

7. Nuestra Raza fue una revista afrouruguaya que circuló durante dos épocas. La primera, en San Carlos, Maldonado, en 1917, editada por los hermanos Ventura y María Esperanza Barrios, a los que se sumará, después, un tercer hermano, el poeta Pilar Barrios. Nuestra Raza. Periódico social, noticioso- Órgano de la colectividad de color. De la raza, por la raza y para la raza circuló desde el 10 de marzo de 1917 hasta el 30 de diciembre de 1917. Para su segunda época, Nuestra Raza fue editada en Montevideo por los hermanos Pilar y Ventura (María Esperanza había fallecido) y circuló desde el 25 de agosto de 1933 hasta septiembre de 1948. Fue la publicación afrouruguaya que más duró, echando raíces invaluables para el movimiento afrouruguayo. Entre las figuras que trabajaron para Nuestra Raza se destacan: Ventura Barrios, Pilar Barrios, Elemo Cabral, Salvador Betervide, Isabelino José Gares, Marcelino Bottaro, Lino Suárez Peña, Selva Escalada, Iris Cabral, Maruja Pereira, Sandalio del Puerto, Mario Rufino Méndez, Felina Díaz, Sandalio Gutiérrez, Carlos Cardozo Ferreira, Mario Leguizamón, Miguel Ángel Bustamante, Julio Guadalupe, Diógenes Rodríguez Barrios, Ildefonso Pereda Valdés y muchos más.

8. Para un entendimiento de la categoría" interseccionalidad", ver Crenshaw 1991.

9. El batllismo en sus primeras épocas (primera mitad del siglo XX) fue una corriente política liberal reformista, en la que el Estado tendía a comportarse como benefactor. Generada a partir de las ideas y acción del dos veces presidente de la república y fundador del diario El Día, José Batlle y Ordóñez.

10. Todas las citas de las publicaciones son textuales, respetando posibles errores ortográficos o de redacción. 
11. En varias partes de la región latinoamericana las mujeres se estaban organizando y tejiendo redes internacionales. Para tener una visión general del movimiento de mujeres en América Latina, ver Valdés 2000.

12. Sobre la propuesta de un sindicato para trabajadoras domésticas, hecha por Iris y Maruja en el congreso, ver: Young 2004, Rodríguez 2006, pp. 120-121; Andrews 2012, 131.

13. Ver los nueve números del P.A.N, desde abril hasta diciembre de 1937.

14. La autopercepción "apática" tiene numerosas referencias en la prensa afrouruguaya. Algunos ejemplos: "Las imposibilidades que en otrora nos sindicaron sumidos en la mayor apatía han ido paulatinamente desapareciendo hasta anularse (La Propaganda 1911, agosto, 10, p. 1); "Los hijos de la apatía son desgraciadamente muchos" (La Verdad 1914, mayo, 15, p. 1); "La apatía de nuestra raza" (Rumbos 1939, junio, p. 1).

15. En muchas ocasiones, a lo largo de toda la producción de la prensa afrouruguaya, desde 1872, se expresó la idea de superación que implicaba dejar atrás un pasado esclavista interiorizado. En las publicaciones del siglo XIX y las primeras del XX, se utilizaba mucho el término "regeneración", connivente con las ideas de modernización y progreso de la época, que para la colectividad afrouruguaya implicaba adoptar nuevos modos culturales y la entrada en un esfuerzo colectivo de afirmación. Ver, entre otros artículos: "Más sobre nuestro voto", La Conservación 1872, octubre, 6, p. 1; “En la unión está la fuerza”, La Propaganda 1894, agosto, 5, p. 1; “Razonar", La Propaganda 1911, junio, $1^{\circ}$, p. 2.

16. A fines de 1937 aún no se sabía si las mujeres votarían. Ver, “¿Vota la mujer?”, de Felina Díaz, P.A.N. 1937, noviembre, 16, p. 3. Aunque la ley del sufragio femenino había sido aprobada en 1932, debido al golpe de Terra, las elecciones presidenciales se derivaron para 1938. Para una historia del voto femenino en el Uruguay, ver Rodríguez 1984.

\section{ABSTRACTS}

Afro-Uruguayan women have participated in various political activities with other women. One of these actions was their presence in the First National Congress of Women of Uruguay, held in Montevideo in 1936. In their representation, the delegates Iris Cabral and Maruja Pereira exposed their proposals and shared all the activities of the meeting. They also connected with other organized women, such as Clementina Gómez who was a member of the Democratic Feminist Party.

The Afro-Uruguayan press covered this participation with the notes of two of its columnists, who highlighted the meritorious action of the "black woman".

In these mentions, from the observation of the press of their community, the intersectionality of Afro-Uruguayan women is notorious as women and as Afro-descendants. The reporters that covered the news of their participation pointed to identity elements of the Afro-Uruguayan women that were different from those of other women in the country.

In this article we will approach the identities of the Afro-Uruguayan woman: her Uruguayan woman side and her "black woman" side. In turn, some of the connections they had with other women will be shown, placing them in their historical context and taking the First National Congress of Women of Uruguay as their main event.

Las mujeres afrouruguayas participaron en varias actividades con otras mujeres a nivel político. Una de estas acciones fue su presencia en el Primer Congreso Nacional de Mujeres del Uruguay 
realizado en Montevideo en 1936. En su representación, las delegadas Iris Cabral y Maruja Pereira expusieron sus propuestas y compartieron todas las actividades del encuentro. Asimismo, se conectaron con otras mujeres organizadas, como Clementina Gómez, miembro del Partido Feminista Democrático.

La prensa afrouruguaya cubrió esta participación con las notas de dos de sus redactores, que destacaron la acción meritoria de la "mujer negra". En estas menciones, a partir de la observación de la prensa de su colectividad, la interseccionalidad de las afrouruguayas es notoria en tanto mujeres y en tanto afrodescendientes. Los redactores, al cubrir la noticia de su participación, señalaron elementos identitarios de las afrouruguayas diferentes a los de las otras mujeres del país.

En este artículo se hará un acercamiento a las identidades de la mujer afrouruguaya: su lado de mujer uruguaya y su lado de "mujer negra". A su vez, se mostrarán algunas de las conexiones que tuvieron con otras mujeres, ubicándolas en su contexto histórico y tomando como acontecimiento principal el Primer Congreso Nacional de Mujeres del Uruguay.

INDEX

Keywords: Afro-Uruguayans, women, congress, intersectionality

Palabras claves: Afrouruguayas, mujeres, congreso, interseccionalidad

\section{AUTHOR}

\section{MÓNICA GARCÍA MARTÍNEZ}

Programa de Posgrado en Estudios Latinoamericanos, Universidad Nacional Autónoma de México, México

monicalatinoamericanos@gmail.com 